\title{
Predictive factors for the recurrence of surgically excised basal cell carcinomas: A retrospective clinical and immunopathological pilot study
}

\author{
CORINA VORNICESCU ${ }^{1}$, SIMONA CORINA ȘENILÄ ${ }^{2,3}$, NONA IONELA BEJINARIU ${ }^{4}$, \\ ȘTEFAN CRISTIAN VESA ${ }^{5,6}$, ADINA BIANCA BOȘCA ${ }^{1}$, DACIANA NARCISA CHIRILA $\breve{~}^{7,8}$, \\ CARMEN STANCA MELINCOVICI ${ }^{1,9}$, OLGA SORIȚĂU $^{10}$ and CARMEN MIHAELA MIHU ${ }^{1,9}$
}

\begin{abstract}
Departments of ${ }^{1}$ Morphological Sciences-Histology and ${ }^{2}$ Dermatology, 'Iuliu Haţieganu' University of Medicine and Pharmacy, 400349 Cluj-Napoca; ${ }^{3}$ Department of Dermatology, Emergency Clinical County Hospital, 400006 Cluj-Napoca; ${ }^{4}$ Department of Pathology, Santomar Oncodiagnostic, 'Regina Maria' Private Healthcare Network, 400664 Cluj-Napoca; ${ }^{5}$ Department of Pharmacology, Toxicology and Clinical Pharmacology,

'Iuliu Hațieganu' University of Medicine and Pharmacy, 400337 Cluj-Napoca; ${ }^{6}$ Department of Internal Medicine, Out-patient's Clinic Infectious Disease Hospital, 400348 Cluj-Napoca; ${ }^{7}$ Department of Surgery, Vth Surgical Clinic,

'Iuliu Hațieganu' University of Medicine and Pharmacy; ${ }^{8}$ Department of Surgery, Clinical Municipal Hospital, 400139 Cluj-Napoca; ${ }^{9}$ Department of Radiology, Emergency Clinical County Hospital, 400006 Cluj-Napoca; ${ }^{10}$ Laboratory of Tumor Biology and Radiobiology, 'Prof. Dr. Ion Chiricuţă' Oncology Institute, 400015 Cluj-Napoca, Romania
\end{abstract}

Received June 29, 2021; Accepted July 29, 2021

DOI: $10.3892 / \mathrm{etm} .2021 .10771$

\begin{abstract}
Basal cell carcinoma (BCC) is the most frequent form of skin cancer and is not a tumor with a lethal outcome if diagnosed and treated adequately. The gold standard for treatment is surgical excision with histologically safe margins. Even so, tumors excised with free margins may recur after a period of time. The identification of predictive factors for the recurrence of BCCs besides the localization, size and aggressive histology may be useful for the clinician. The aim of the present study was to identify clinical and pathological factors associated with recurrence in tumors with histologically free margins and assess via immunohistochemical staining, the expression of glioma-associated oncogene homolog 1 (GLI1), yes-associated protein (YAP), connective tissue growth factor (CTGF) and E-cadherin as they are involved in the development of BCCs, in the hope of identifying markers
\end{abstract}

Correspondence to: Dr Simona Corina Șenilă, Department of Dermatology, Emergency Clinical County Hospital, 3-5 Clinicilor Street, 400006 Cluj-Napoca, Romania

E-mail: corina.senila@umfcluj.ro

Abbreviations: BCC, basal cell carcinoma; MMS, Mohs micrographic surgery; Hh, Hedgehog pathway; GLI1, gliomaassociated oncogene homolog 1; YAP, yes-associated protein; CTGF, connective tissue growth factor; NMSC, non-melanoma skin cancer

Key words: basal cell carcinoma, recurrent tumors, GLI1, YAP, CTGF, E-cadherin that are predictive for recurrence. In total, 8 recurrent BCCs and 38 non-recurrent tumors were analyzed. A Breslow index $>2(\mathrm{Se} 100.0 \%, \mathrm{Sp} 67.5 \%, \mathrm{P}=0.008)$, Clark level $>3$ (Se 100.0\%, Sp 47.5\%, $\mathrm{P}<0.001$ ), and excision margins both lateral (Se 87.5\%, Sp 60.0\%, P=0.04) and deep (Se 75.0\%, Sp $82.5 \%, \mathrm{P}<0.001)$ free from tumoral cells $\leq 1 \mathrm{~mm}$ proved to be predictive for recurrence in the present study. Recurrence may appear even after more than 3 years since the initial excision ( $\mathrm{Se} 87.50 \%, \mathrm{Sp} 70.0 \%, \mathrm{P}<0.001$ ). The expression levels of GLI1, YAP and E-cadherin were not different in the recurrent vs. non-recurrent BCCs. However, the low expression of CTGF may indicate a tumor with a higher aggressiveness. In conclusion, close follow-up of patients with excised BCCs at least annually is recommended and re-excision should be taken into consideration for locally advanced tumors especially if they are located in high-risk areas or those with histologically free margins $<1 \mathrm{~mm}$.

\section{Introduction}

Basal cell carcinoma (BCC) is the most frequent form of skin cancer and it is characterized by slow progression and eventually local destruction. Usually, BCC does not have a lethal outcome, if associated with proper treatment (1). According to recent findings, the risk of a fair-skin person developing at least one BCC is $30 \%$ (1). Moreover, an individual may have multiple BCCs or a BCC associated with other sun-related skin neoplasia, and some lesions may recur after treatment (2). Consequently, in countries with mostly light-skinned individuals, the incidence of BCC is extremely high, which leads to proportionately increasing burden on the medical system, both economically and logistically, due to the need for regular screening. 
The diagnosis of BCC is based on clinical findings and paraclinical investigations, mainly the pathological exam. Dermoscopy increases the sensitivity to $91.2 \%$ and specificity to $95 \%$ of the diagnosis in $\mathrm{BCC}$, due to specific dermatoscopic features (1). Additional diagnostic elements can be provided by confocal microscopy (3) and ultrasound examination (4).

Localization and tumor size are important risk factors for recurrence (5). They contribute to its characterization as high- vs. low-risk tumor. The concept of the $\mathrm{H}$ zone (high risk for recurrence zone) comprises: Nasolabial fold, nasal, orbital and auricular areas (5). Another factor that has been attributed to higher recurrence rates is the presence of positive histological margins, which is more common in the head area (6). Tumors that have even partially aggressive histologic growth patterns such as micronodular, infiltrative, and sclerodermiform are more likely to recur compared to nodular of superficial subtypes (5). Basosquamous BCC is a special category which has higher recurrence risk and metastasis rates, comparable to squamous cell carcinoma, rather than with BCC (7).

The gold standard of treatment in BCC is surgical excision with tumoral-free margins. This can be achieved by classical surgery or by Mohs micrographic surgery (MMS). For patients with locally advanced BCCs or with metastasis [which are very uncommon: $0.0028-0.55 \%$ (1)] that cannot be successfully treated using surgical methods, radiotherapy or systemic medication are recommended: Hedgehog inhibitors (vismodegib and sonidegib) (1).

Classical surgery consists of the removal of the tumor and variable amount of the apparently healthy surrounding tissue, with a recurrence rate of $2-8 \%$ at 5 years (8). Clinically free margins should be selected as follows: i) for tumors with low risk of recurrence: $3-4 \mathrm{~mm}$; ii) for high-risk tumors, when MMS is not available: $5-15 \mathrm{~mm}$; iii) in depth, the excision should reach the subcutaneous fat, or, for cervicofacial area, the excision should be extended to the muscular fascia, perichondrium or periosteum (1).

MMS is a more expensive and time-consuming surgical technique. However, this technique allows for a more complete analysis of the margins, and thus enables better preservation of the surrounding healthy tissue. MMS is recommended for tumors of the face especially in the $\mathrm{H}$ zone, recurrent tumors or tumors with aggressive histological features. The reported recurrence rate is $3.9 \%$ after 10 years (9).

However, not all dermatologic services have access to this performant treatment modality. Consequently, doctors may use classical surgery even for tumors in high-risk areas or with an aggressive phenotype.

The pathogenesis of BCC is incompletely elucidated. Many molecules, growth factors and adhesion molecules are involved. Disruptions of the Hedgehog pathway (Hh) are the most common alterations in the development of BCC. The signature molecule of $\mathrm{Hh}$ is glioma-associated oncogene homolog 1 (GLI1). The most studied activation pathway of $\mathrm{Hh}$ is through the glycoprotein sonic $\mathrm{Hh}(\mathrm{SHH})$ and this activation pathway has an important role in the embryological development of various tissues and organs and in maintaining cell polarity (10). It was firstly isolated in a glioma, thus the name of glioma-associated oncogene. Abnormal activation of SHH was reported in different malignancies such as BCC, medulloblastoma, leukemia, and carcinomas of the breast, lung, pancreas and prostate (10).

Yes-associated protein (YAP) is essential for the stem cell population and for BCC proliferation. YAP is a transcriptional factor of the Hippo signaling pathway, which controls organ size and tumor inhibition (11). The activation of Hippo leads to the phosphorylation of YAP and its retention in the cytoplasm. In the absence of Hippo inhibition, YAP, together with the transcriptional coactivator with PDZ-binding motif (TAZ) are translocated to the nucleus where they induce the expression of genes responsible for proliferation, cell growth, epithelial-mesenchymal transformation and the inhibition of apoptosis (12).

YAP's interaction with the surrounding microenvironment is through connective tissue growth factor (CTGF) (13). CTGF, also known as CCN2, is part of the CCN family of proteins. The main purpose of CTGF is to promote cell adhesion through heparin and other integrins depending on tissue (14). CTGF also has a role in migration processes, cell proliferation, angiogenesis, tissue repairing, fibrosis and others (15).

E-cadherin belongs to the cadherin group of molecules responsible for calcium-dependent cell-to-cell adhesion. The intercellular adhesions are mediated by the extracellular domains of these cadherins, while their intracellular part is bound to numerous adaptor or signaling proteins. YAP is regulated by the surrounding factors through E-cadherin (16).

Patients with excised BCCs with positive histological margins may subsequently undergo re-excision, radiotherapy or adequate clinical assessment and in some cases an ultrasound exam of the surgical scar. However, patients with tumoral-free margins are probably less attentively followed up and, in case of recurrence, may present locally advanced BCCs, which are more difficult to treat. In addition, patients with a history of BCC may develop a second BCC or multiple BCCs, some of which may be less compliant to the use of photoprotection. Thus, the identification of additional predictive markers for recurrence also becomes an important concern in skin cancer prevention.

The aim of the present study was to assess the clinical and histopathological characteristics of the tumors that become recurrent after classical surgery with initially free histologic margins. In addition, the expression of GLI1, YAP, CTGF and E-cadherin was assessed as they have an impact in the development of BCC and in the attempt to find histological markers that may be useful for the identification of possible future recurrences after an initial excision with tumoral-free margins.

\section{Patients and methods}

Participants. A retrospective observational study in the Department of Dermatology, Emergency Hospital (Cluj-Napoca, Romania) was performed. Ethics committee approval (under the number 155/07.04.2017) was obtained as well as informed consent from the participants. The study included patients whose initial BCCs were excised with free histological margins, who were readmitted and underwent a second excision between January 2012 and October 2015 for a recurrence in the Department of Dermatology. The patients were selected from the 961 admissions for non-melanoma 


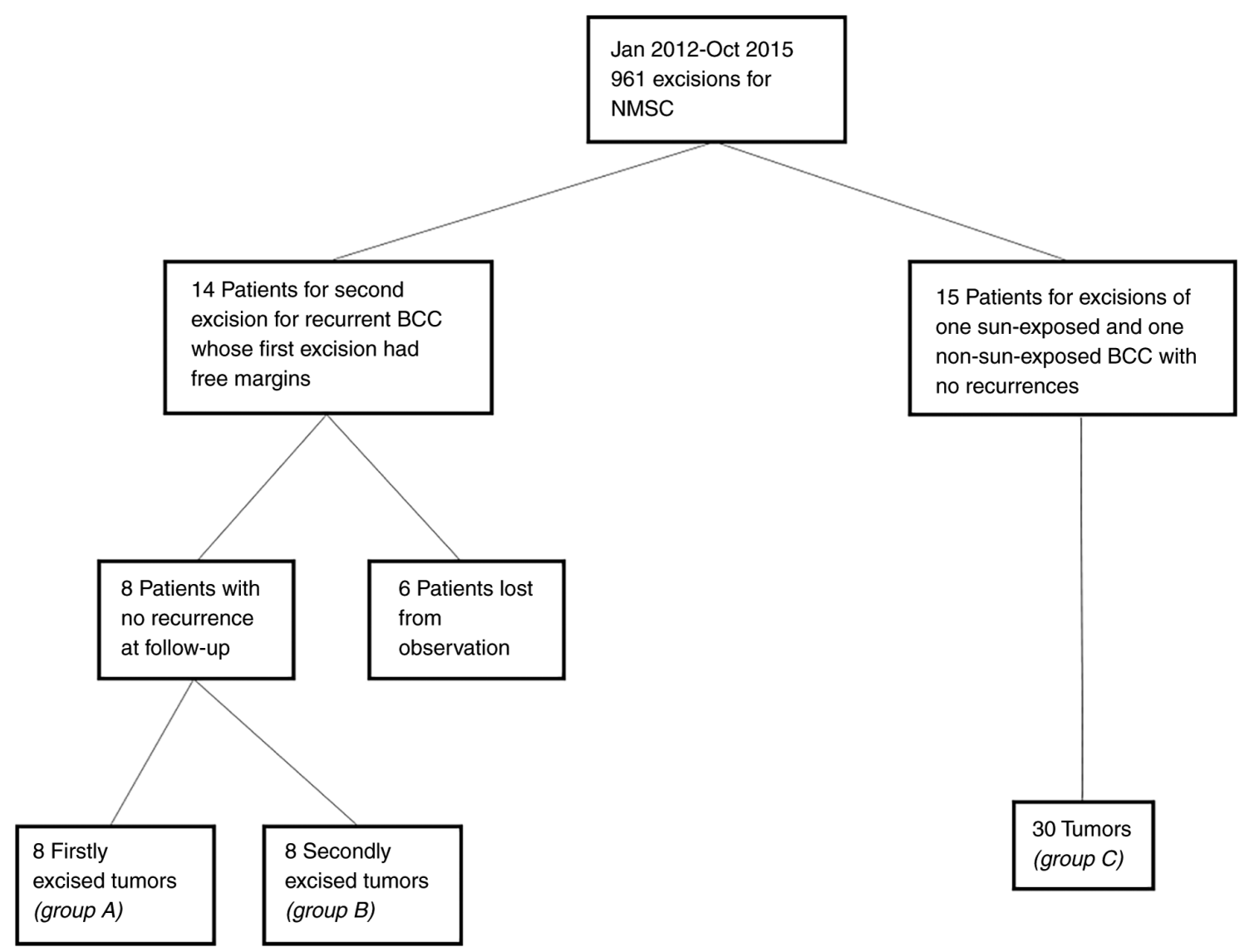

Figure 1. Selection of the study patients/tumors.

skin cancer (NMSC) excisions, according to a methodology described in a previously published study (17) (Fig. 1).

Inclusion and exclusion criteria. The inclusion criteria were: i) Patients who underwent classical excision for the first recurrence of a previously excised BCC; ii) patients whose initially excised tumor had free histological margins. The exclusion criteria were: i) patients with squamous cell carcinoma or benign tumors; ii) patients with initial or recurrent tumor with at least one positive histological margin; iii) patients with initial or recurrent tumor of basosquamous subtype or with perineural invasion; iv) patients treated by Mohs surgery; v) patients who presented re-recurrence after the second excision; vi) patients who did not present for follow-up between November 2017 and November 2018. A total of 14 patients met the inclusion criteria, while 6 patients were excluded. From the remaining 8 patients the initial BCCs (group A: Recurred tumors, case group) and the recurrent BCCs (group B: Non-recurred tumors, part of the control group) were analyzed.

Additionally, the control group included tumors from patients with multiple BCCs, with the following inclusion criteria: i) patients who underwent surgery for both a tumor in a sun-exposed area (nose, paranasal area, ear, preauricular area, the rest of the face, neck, scalp, anterior and posterior thorax, superior limbs) and a tumor in a non-sun-exposed area (retroauricular area, inferior limbs, abdomen, lumbar zone, genitalia) concomitantly or in consecutive steps; ii) patients who underwent both surgeries between January 2012 and October 2015. The exclusion criteria were: i) patients who presented recurrence at follow-ups; ii) patients treated by Mohs surgery; iii) patients with tumors of basosquamous subtype or with perineural invasion; iv) patients who had more than two BCCs excised; v) patients who did not present for follow-up in November 2017 to November 2018. From 961 admissions, 15 patients with 30 tumors (group C) met the inclusion criteria.

Factors and classification. For the selected tumors, the following characteristics were recorded: time passed from the initial excision (years), time until recurrence appearance (years), localization, dimension $(\mathrm{cm})$, main morphology ranked according to aggressiveness: infiltration (also including the micronodular subtype), sclerodermiform, nodular, superficial, cystic. Additionally, the Breslow index and Clark level were recorded, as well as the extension of tumoral cells in lateral and deep margins (mm).

Taking into consideration their location, tumors were divided into one of three risk localization categories: i) high risk: nose and paranasal (including the nasolabial fold), auricular and periauricular; ii) medium risk: rest of the face, neck, scalp; iii) low risk: trunk, limbs. Regarding the risk of recurrence, the tumors were divided in 2 categories: i) high risk (tumors with high risk localization, or medium risk localization and dimension $\geq 1 \mathrm{~cm}$, or low risk localization and dimension $\geq 2 \mathrm{~cm}$, or histologically diagnosed as infiltrative or sclerodermiform); or ii) low risk: the other types of tumors.

Immunohistochemistry. For the immunohistochemical analysis, the selected tumors were formalin-fixed, 


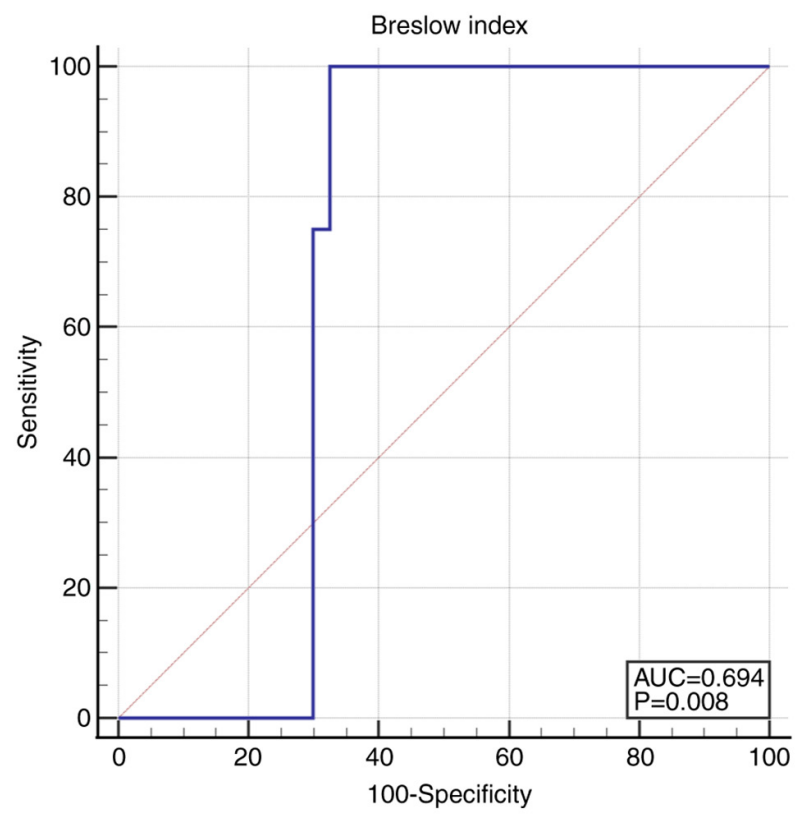

Figure 2. ROC curve for Breslow index $>2.0015$. ROC, receiver operating characteristic.

paraffin-embedded and 5- $\mu \mathrm{m}$ tissue sections were used in the most representative areas, where the malignant tumor was predominant. The sections were stained with antibodies for E-cadherin (Clone EP700Y; Roche, Ventana; BenchMark Ultra, CC1 standard, 16 min incubation with primary antibody, OptiView amplification); anti-YAP (rabbit monoclonal EP1675Y' Abcam, 1:200 dilution), GLI1 (rabbit polyclonal; Thermo Fischer Scientific, Inc, $5 \mu \mathrm{g} / \mathrm{ml}$ ), CTGF/CCN2 (rabbit polyclonal' Novus Biologicals; 1:50 dilution) and the slides were analyzed under x100 and x400 magnification, using an Olympus microscope BX43. Immunostaining was assessed by the same pathologist both as a percentage (with an average of $5 \mathrm{HPF})$ and staining intensity (0, none; 1 , low; 2 , medium; or 3 , high).

Statistical analysis. Statistical analysis was performed using the MedCalc ${ }^{\circledR}$ Statistical Software version 19.6 (MedCalc Software Ltd., Ostend, Belgium; https://www.medcalc.org; 2020). Quantitative variables were tested for normality of distribution using the Shapiro-Wilk test and were expressed as median and 25-75 percentiles. Qualitative variables were characterized by frequency and percentage. Comparisons between groups were carried out using the Mann-Whitney test or Chi-square test, whenever appropriate. The area under the receiver operating characteristic (ROC) was used to identify the ability of a quantitative variable to predict the recurrence. $\mathrm{P}<0.05$ was considered to indicate statistical significance.

\section{Results}

In total, 8 recurrent tumors (group A) and 38 non-recurrent tumors (groups B and C) were clinically and immunopathologically assessed. Due to the small number of patients with recurrent tumors, the comparison between recurrent and non-recurrent tumors harvested from the same patient was not possible. None of the 23 patients had metastatic BCC.
Results of clinical and pathological assessment. The tumors were situated predominantly in the head and neck: 28 tumors (60.9\%), compared with the trunk and abdomen: 17 tumors $(36.9 \%)$ and only one $(2.2 \%)$ tumor was located on the extremities $(\mathrm{P}=0.45)$ (Table I). All the recurrent tumors were located in the head and neck areas. Regarding the categories of risk according to localization, $62.5 \%$ of recurrences occurred in high-risk zones, $37.5 \%$ in medium-risk zones, and none in low-risk zones. By contrast, non-recurrent tumors were evenly distributed in the three categories: 9 (23.7\%) high risk, 11 $(28.9 \%)$ medium risk, $18(47.4 \%)$ low risk $(\mathrm{P}=0.04)$.

The macroscopic size of the tumors was similar in both groups $1.0 \mathrm{~cm}(0.63-1.38)$ for recurrences and $0.9 \mathrm{~cm}(0.6-0.9)$ for non-recurrent tumors $(\mathrm{P}=0.82)$.

Morphologically, recurrent tumors were $50 \%$ nodular and $50 \%$ infiltrative. Non-recurrent tumors were: 31 nodular (81.6\%), 2 infiltrative (5.3\%), 3 superficial (7.9\%), 1 sclerodermiform $(2.6 \%)$, and 1 cystic $(2.6 \%)(\mathrm{P}=0.08)$. Pigmentation was found in $15.8 \%$ of non-recurrent tumors, while none of the recurrent tumors showed pigmentation $(\mathrm{P}=0.57)$. Ulceration was found in $6(75 \%)$ recurrent tumors compared to $23(60.5 \%)$ primary tumors $(\mathrm{P}=0.12)$.

All recurrent tumors were in the category of high risk for recurrence, whereas 20 non-recurrent tumors (52.6\%) were also in the high-risk category $(\mathrm{P}=0.01)$ (Table $\mathrm{I})$.

Breslow index was significantly lower in non-recurrent tumors, 1.8 (1.0-2.5) compared to $2.2(2.1-2.3)$ in recurrent tumors $(\mathrm{P}=0.05)$. The cut-off value for recurrence of Breslow index was higher than 2.0015: AUC 0.694 (95\% CI 0.544-0.819), Se 100.0 (95\% CI 63.1-100.0), Sp 67.5 (95\% CI 50.9-81.4), $\mathrm{P}=0.008$ (Fig. 2).

The Clark level was also significantly lower in non-recurrent tumors 4 [3-4] compared to 4 [4-4] in recurrent tumors $(\mathrm{P}=0.02)$. For the Clark level a cut-off value of 3 was calculated, above which the risk of recurrence increased: AUC 0.733 (95\% CI 0.585-0.850), Se 100.0 (95\% CI 63.1-100.0), Sp 47.5 (95\% CI 31.5-63.9), $\mathrm{P}<0.001$ (Fig. 3).

The lateral margins were more distant from the tumor in non-recurrent tumors $1.09 \mathrm{~mm}(1.0-2.0)$ compared to recurrent tumors $0.95 \mathrm{~mm}(0.13-1)(\mathrm{P}=0.05)$. The cut-off value under which the recurrence rate increased was $1 \mathrm{~mm}$ : AUC 0.716 (95\% CI 0.567-0.836), Se 87.5 (95\% CI 47.3-99.7), Sp 60.0 (95\% CI 43.3-75.1), $\mathrm{P}=0.04$ (Fig. 4).

The deep margin was 2-fold deeper in non-recurrent tumors, $1.85 \mathrm{~mm}(1.2-2.6)$ compared to $0.8(0.2-1.4)$ in the recurrent tumors $(\mathrm{P}=0.009)$. The cut-off value was $1 \mathrm{~mm}$, under which the recurrence rate increased: AUC 0.795 (95\% CI 0.654-0.898), Se 75.0 (95\% CI 34.9-96.8), Sp 82.5 (95\% CI 67.2-92.7), $\mathrm{P}<0.001$ (Fig. 5).

The median time from the primary tumor excision to recurrence appearance was 2 years (1.0-4.0).

Results of the immunohistochemical analysis. The immunostaining intensity for GLI1 (Fig. 6) was scored 0 in $21.7 \%$ of all tumors, 1 in $58.7 \%$, and 2 in $19.6 \%$ of tumors. None of the tumors showed score 3 . GLI1 expression had an intensity of 1 to 2 in $75 \%$ of recurrent tumors and in $78.9 \%$ of non-recurrent tumors $(\mathrm{P}=0.89)$. The percentage of positive cells was similar, with a median at $10 \%$ in both recurrent and non-recurrent tumors (Table II) $(\mathrm{P}=0.84)$. 


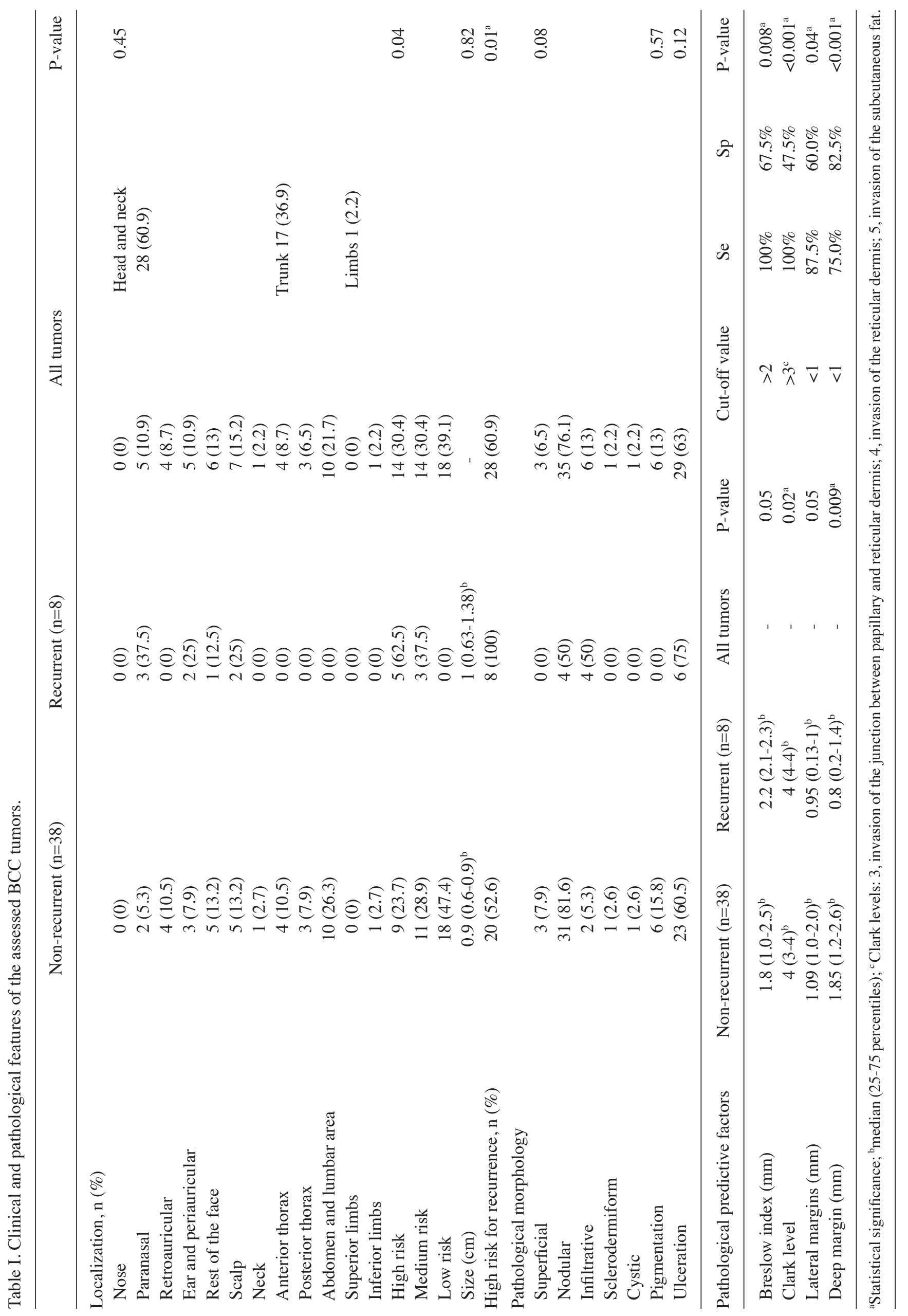




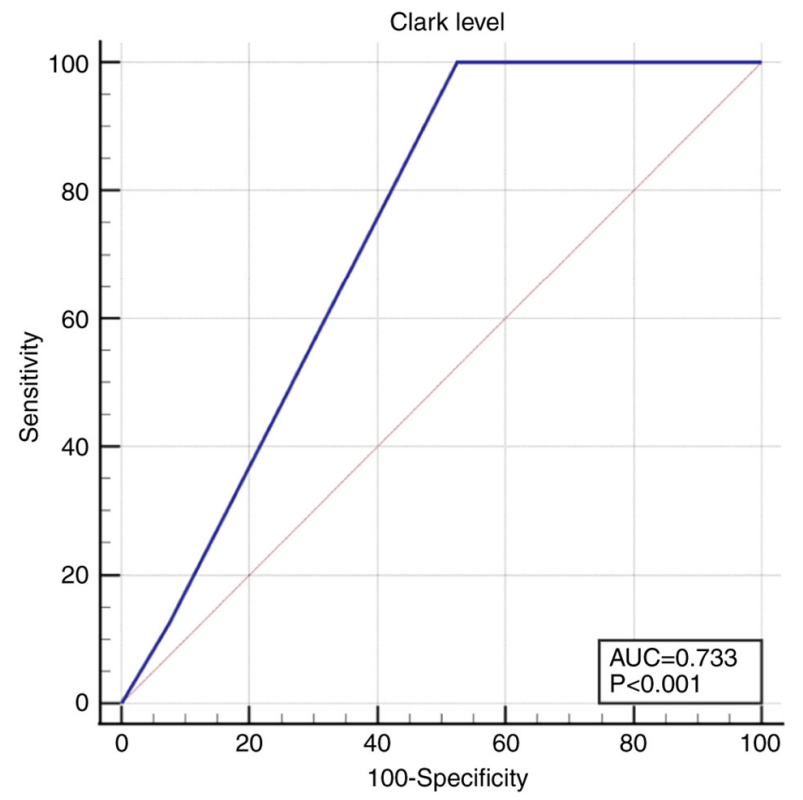

Figure 3. ROC curve for Clark level $>3$. ROC, receiver operating characteristic.

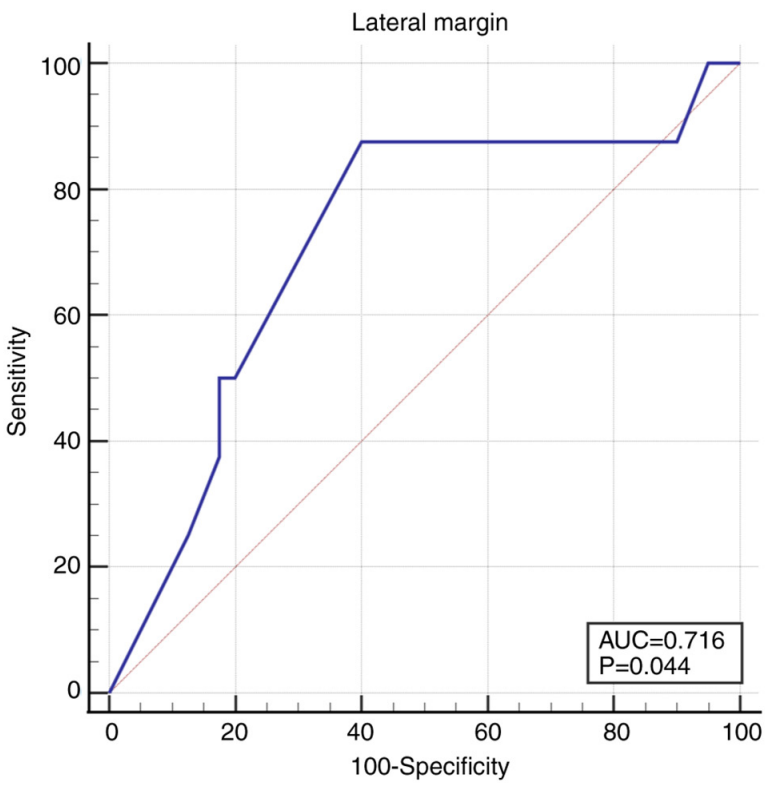

Figure 4. ROC curve for lateral margins $<1 \mathrm{~mm}$. ROC, receiver operating characteristic.

All tumors were immunopositive for YAP (Fig. 7). The immunostaining intensity for YAP was 1 in $10.9 \%$ of all tumors and 2 to 3 in $89.1 \%$ of all tumors. There was no difference between recurrent and non-recurrent tumors; all recurrent tumors and $86.8 \%$ of the non-recurrent tumors had an intensity of 2 to $3(\mathrm{P}=0.55)$. YAP was expressed in most cells, both in recurrent and non-recurrent tumors, at a percentage of $80 \%$ (70-100) and 100\% (80-100) respectively, with no statistical significance (Table II) $(\mathrm{P}=0.31)$.

All tumors were immunopositive for CTGF (Fig. 8). The intensity score was 1 to 2 in $80.4 \%$ of all tumors and 3 in $19.6 \%$ of tumors. CTGF was less expressed in recurrent tumors compared to the non-recurrent tumors. The intensity score was 1 in $62.5 \%$ of recurrences vs. $21 \%$ of non-recurrences, and

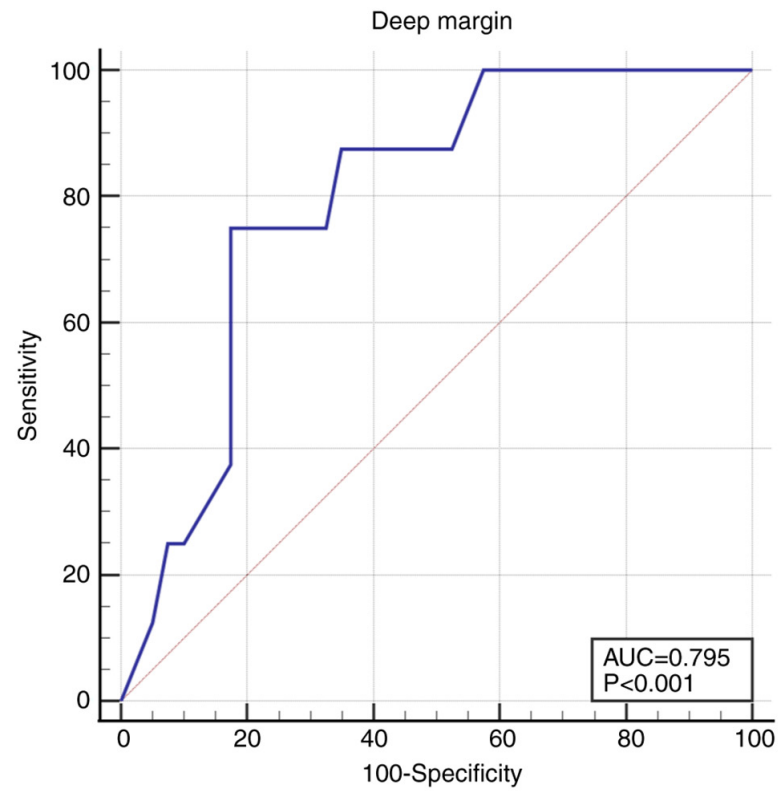

Figure 5. ROC curve for deep margin $<1 \mathrm{~mm}$. ROC, receiver operating characteristic.

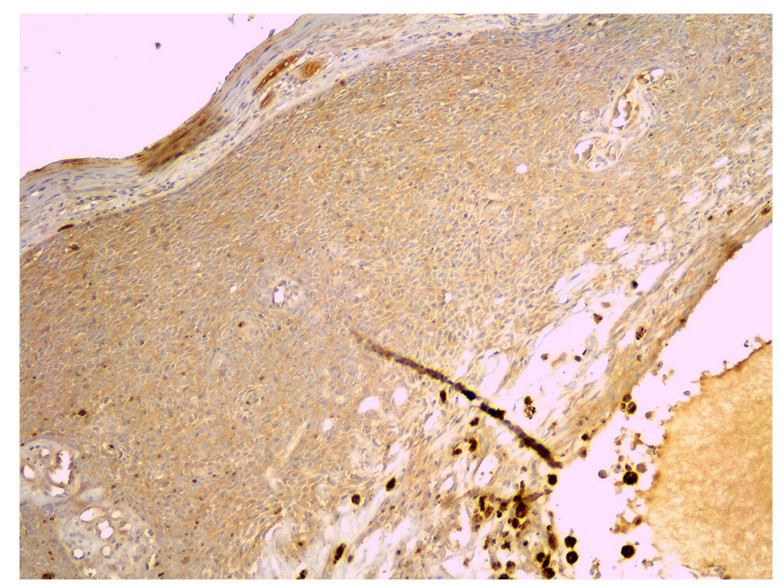

Figure 6. Immunostaining for GLI1, x40 magnification. GLI1, gliomaassociated oncogene homolog 1 .

score 2 in $37.5 \%$ of recurrences vs. $55.3 \%$ of non-recurrences $(\mathrm{P}=0.05)$. Regarding the percentage of immunostained cells, CTGF was expressed less in recurrent tumors, $20 \%$ (6.25-78.75) compared to non-recurrent tumors, $75 \%$ (30-90) (Table II) $(\mathrm{P}=0.09)$.

The staining intensity for E-cadherin (Fig. 9) was 0 in only one tumor (2.2\%). Scores 1 and 2 were found in $84.8 \%$ of tumors. A score of 3 was found in $13 \%$ of all tumors. The intensity score 3 was found in $15.8 \%$ of the non-recurrent tumors, but in none of the recurrent tumors $(\mathrm{P}=0.57)$. The percentage of E-cadherin-positive cells was 15\% (10-28.75) in recurrent tumors and 20\% (10-40) in non-recurrent tumors (Table II) $(\mathrm{P}=0.59)$.

\section{Discussion}

$\mathrm{BCC}$ is a cutaneous tumor with slow progression and complete cure when treated adequately. The gold standard of treatment 


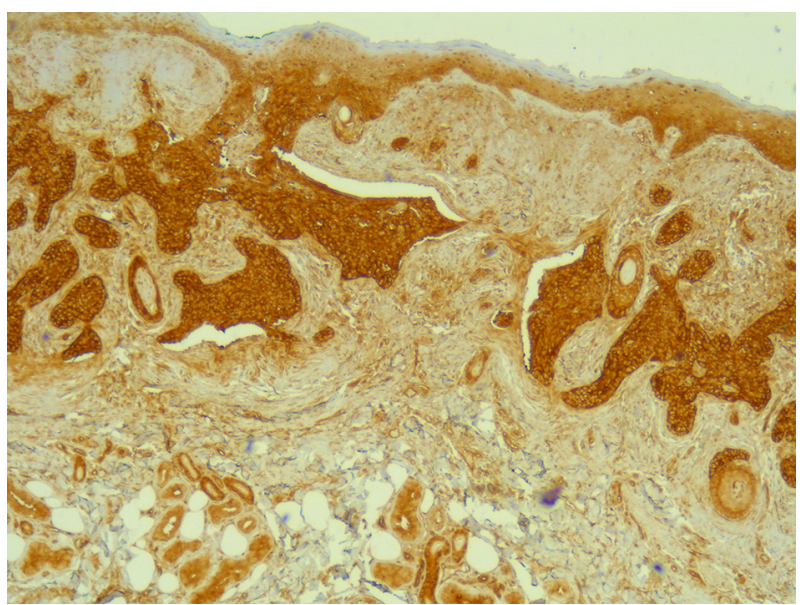

Figure 7. Immunostaining for YAP, x40 magnification. YAP, yes-associated protein.

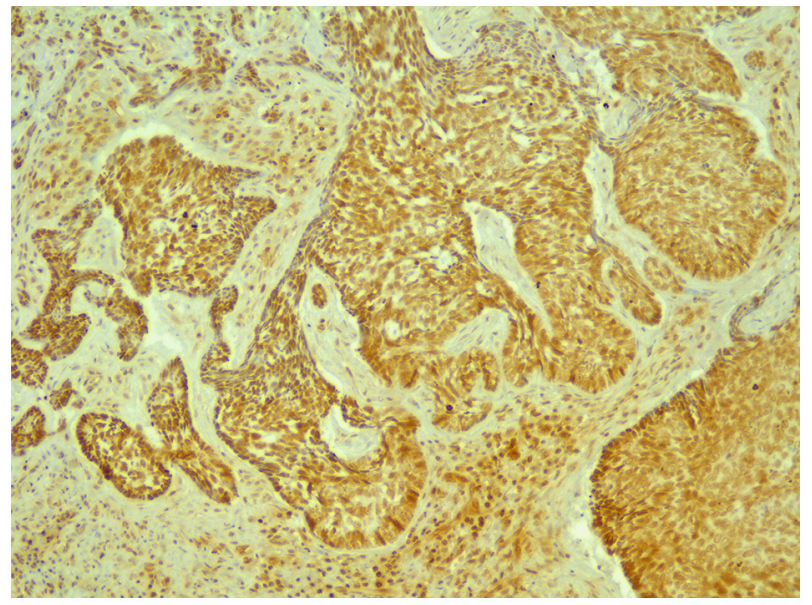

Figure 8. Immunostaining for CTGF, $x 40$ magnification. CTGF, connective tissue growth factor.

in $\mathrm{BCC}$ is surgical excision. For high-risk BCCs such as those located in critical anatomical areas, recurrent BCCs, or large tumors, MMS is recommended as it allows a much more precise evaluation of resection margins (18).

The aim of the current study was to identify clinical and pathological predictive factors for the risk of recurrence of BCCs excised with negative histological margins especially those located in high-risk areas, because these patients might be less compliant to the long-term follow-up.

The results showed that, the pathological predictive factors for the risk of recurrence were: Breslow index $>2$, Clark level $>3$, and lateral or deep margins closer than $1 \mathrm{~mm}$. Results of the present study are supported by Girardi et al who considered, in their study, that the positive margins or submilimetrical negative margins were inadequate. They also showed that the Clark level and the depth of invasion (Breslow index) were independent risk factors for insufficiently safe margins. Thus, in their study, tumors with inadequate margins had a Clark level of 4 to 5 , and the median of Breslow index was at 2, similar to our results (19).

The European guideline for BCC 2019 does not clearly define the optimal histological margins and it uses terms such 


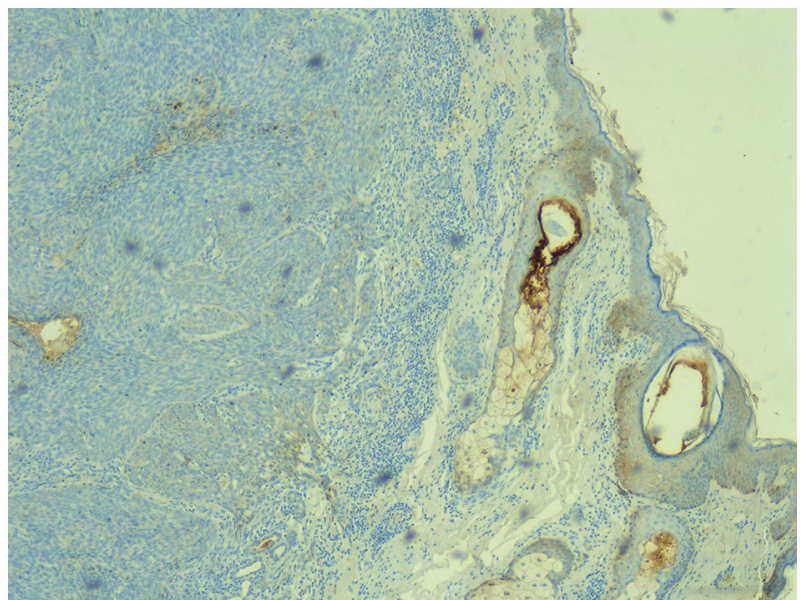

Figure 9. Immunostaining for E-cadherin, x40 magnification.

as 'incompletely excised' and 'not optimal' (1). Our results showed that lateral or deep margins narrower than $1 \mathrm{~mm}$ increase the risk of recurrence of excised BCCs. From our point of view, limited by the small number of patients included in the study, it is deemed that free margins of $1 \mathrm{~mm}$ or more would decrease this risk.

Most recurrences appear within the first 3 years after the initial excision, most of them being located in the head and neck area (20). This observation supports the period of time chosen for follow up of the patients at a minimum 3 years after the excision. All the recurrent tumors in our study were located in the head and neck area as expected, similar to the results reported by Bartoš et al (20) and Saabye Bøgelund et al (21). In the current case, the median time of recurrence was 2 years; however, in some of the studied cases tumors recurred even after more than 3 years. Thus, patients diagnosed with a BCC, even though it was excised with histologically free margins should be evaluated at least annually for the remainder of patients' lives to diagnose other metachronous malignant skin tumors that may occur and to advise on photoprotection.

Other than clinical and histopathological parameters observed in hematoxylin and eosin staining, we assessed the influence of some molecules that are studied for their involvement in the carcinogenesis of BCC but not yet related to their clinical behavior. The aim was to evaluate their predictive value regarding the risk of recurrence after surgical excision. There were no statistically significant differences between tumors that recurred and tumors that did not recur, but some important differences were found for CTGF.

Few data exist in the literature regarding the role of GLI1 in cutaneous carcinogenesis. Its implication in the recurrence of BCC is not known. The development of BCCs and other hair follicle-derived tumors (trichoepiteliomas, cylindromas, trichoblastomas) was observed in transgenic mice with enhanced GLI1 expression in the basal layer of the epidermis and in the hair follicle. This suggests that overexpression of GLI1 is sufficient for tumor formation (22). The current results showed that GLI1 had a low-to-medium expression both in recurrent and non-recurrent tumors, which confirms the abnormal activation of $\mathrm{Hh}$ in BCCs. With the limits of a pilot study, with a reduced number of patients that does not permit statistical significance, GLI1 may have a role in the recurrence of tumors as indicated in studies on other types of cancer. The expression of GLI1 in squamous carcinomas of the lung was associated with lymphatic metastasis and poor prognosis (23). Additionally, inhibition of $\mathrm{Hh}$ reduced tumor progression and recurrences in small cell lung cancer (24).

The role of YAP in the pathogenesis of BCC remains to be sufficiently elucidated. Previous findings showed that in BCC, YAP is essential for tumor progression. In transgenic mice, the deletion of YAP and TAZ resulted in the prevention of tumor formation (25). Our results indicated a medium-high expression of YAP, throughout the entire tumor. The limited data of the present study support the essential role of YAP for tumor progression. Additionally, overexpression of YAP was correlated with poor prognosis in patients with hepatocellular (26), mammary (27) and prostatic carcinoma (28).

In BCC, after YAP activation, the tumor stroma is remodeled through CTGF (13). The role of CTGF in carcinogenesis and oncogenic behavior is controversial. The high expression of CTGF was associated with poor prognosis in ovarian epithelial carcinoma (29). However, a low expression of CTGF was associated with lymphatic metastasis, recurrences and low survival rate in colorectal cancer (30). In the present study, this marker had a low-medium overall expression which may explain the low metastatic rate of BCC knowing the dependence of tumoral cells to the surrounding stroma. In this study, CTGF had a tendency to be lower both as intensity of staining and percentage of positive cells in tumors that eventually recurred compared to tumors that did not recur, without significant differences.

The role of E-cadherin in BCC was more studied than that of GLI1, YAP or CTGF. Some authors reported the decrease of E-cadherin expression in morpheaform and recurrent BCCs compared to nodular, cystic and superficial subtypes (31). In the present study, E-cadherin was expressed at low-medium levels in most of the tumors. The lower expression in recurrent tumors, even without statistical significance is similar to previously published studies. Its low expression is an indicative of aggressiveness in other epithelial carcinomas. The expression of E-cadherin was decreased at the tumor invasive front and also in recurrences and metastasis of oral squamous cell carcinoma (32). A meta-analysis reported that reduced or dysfunctional E-cadherin expression was correlated with poor prognosis in head and neck squamous cancers (33). Moreover, in urothelial carcinoma, the low expression of E-cadherin was related to a high risk of recurrences (34).

Although, in most cases, BCC is not an aggressive tumor with a life-threatening potential it is important for it to be diagnosed and treated appropriately. In Romania, the addressability of the population to medical services for the diagnosis and treatment of BCC is growing. This also increases the number of surgical excisions, especially through classical surgery. For tumors with high risk of recurrence, the patients are referred to medical centers which have access to MMS. An attentive follow-up after the excision, even if the resection margins are free form tumoral cells, is important both for the patient and for the physician. This underlines the need for tools as predictive factors to estimate the risk of recurrence. If for a patient with positive histological margins, the doctor's 
attention is obviously present, patients with tumoral-free margins should not be forgotten. We identified Breslow index over 2, Clark level over 3, lateral or deep margins below $1 \mathrm{~mm}$ as predictive factors for $\mathrm{BCC}$ recurrence and studied the immunostaining of GLI1, YAP, CTGF and E-cadherin as they are regarded as being important in carcinogenesis.

The study's limitations include the low number of cases analyzed because most recurrences appear in BCCs with positive histological margins. Moreover, the number of cases was limited due to patients who did not present for follow up, hence the impossibility of assessing the postoperative scar. For this reason, follow up visits are crucial both for the dermatologist and patient.

In summary, in the context of increasing incidence of $\mathrm{BCC}$ in the fair-skinned elderly population, it is important to have tools as predictive factors for the behavior of the tumors. Locally advanced tumors, with Clark level $>3$ and Breslow index $>2$ have an increased risk to become recurrent. Additionally, the risk of recurrence is increased in tumors with submilimetrical histologic margins. Thus, re-excision of such tumors, especially if they are in the high-risk category must be taken into consideration. Follow-up should continue even after the 3 years recommended in the current literature. In our opinion, observational screening is important concerning both the identification of recurrences and the diagnosis of other possible skin neoplasia.

In the future, immunohistochemical analysis resulted from molecules implicated in the development and progression of BCC may be employed as a supplementary tool for evaluation of the aggressiveness and the risk of recurrence. In the current pilot study, GLI1, YAP and E-cadherin did not prove to be important in predicting the recurrence of BCC. The low expression of CTGF potentially indicates a tumor with higher aggressiveness.

\section{Acknowledgements}

Not applicable.

\section{Funding}

The study was partially supported by the project PCD 1300/72/13 January 2017 of 'Iuliu Hațieganu' University of Medicine and Pharmacy Cluj-Napoca.

\section{Availability of data and materials}

The datasets used and/or analyzed during the current study are available from the corresponding author on reasonable request.

\section{Authors' contributions}

CV, NIB, SCS and CMM contributed to the design of work. $\mathrm{CV}$, SCS, ABB, DNC, CSM and CMM were responsible for literature search and manuscript preparation. CV, SCS, NIB, SCV, AAB, DNC, CSM, OS and CMM contributed to design of study, data collection, literature search, manuscript preparation, and critical revision of manuscript for important intellectual content. CV and SCS confirm the authenticity of all the raw data. All authors read and approved the final version of manuscript.

\section{Ethics approval and consent to participate}

Ethics committee approval was obtained from the 'Iuliu Hatieganu' University of Medicine and Pharmacy Cluj-Napoca (approval no.: 155/07.04.2017). All the participants signed an informed consent.

\section{Patient consent for publication}

Not applicable.

\section{Competing interests}

The authors declare that they have no competing interests.

\section{References}

1. Peris K, Fargnoli MC, Garbe C, Kaufmann R, Bastholt L, Seguin NB, Bataille V, Marmol V del, Dummer R, Harwood CA, et al: Diagnosis and treatment of basal cell carcinoma: European consensus-based interdisciplinary guidelines. Eur J Cancer 118: 10-34, 2019.

2. Verkouteren JAC, Ramdas KHR, Wakkee M and Nijsten T: Epidemiology of basal cell carcinoma: Scholarly review. Br J Dermatol 177: 359-372, 2017.

3. Weedon D: Tumors of the epidermis. In: Skin Pathology, 3rd edition. Elsevier, pp668-703, 2010.

4. Wortsman X: Sonography of facial cutaneous basal cell carcinoma. J Ultrasound Med 32: 567-572, 2013.

5. National Comprehensive Cancer Network (NCCN): Basal Cell Skin Cancer (version 1.2020). Available from: https://www.ncen. org/professionals/physician_gls/pdf/nmsc.pdf.

6. Lara F, Santamaría JR and Garbers LE: Recurrence rate of basal cell carcinoma with positive histopathological margins and related risk factors. An Bras Dermatol 92: 58-62, 2017.

7. Lima NL, Verli FD, De Miranda JL and Marinho SA: Basosquamous carcinoma: Histopathological features. Indian J Dermatol 57: 382-383, 2012.

8. Trakatelli M, Morton C, Nagore E, Ulrich C, Del Marmol V, Peris K and Basset-Seguin N; BCC subcommittee of the Guidelines Committee of the European Dermatology Forum: Update of the European guidelines for basal cell carcinoma management. Eur J Dermatol 24: 312-329, 2014.

9. Van Loo E, Mosterd K, Krekels GA, Roozeboom MH, Ostertag JU, Dirksen CD, Steijlen PM, Neumann HA, Nelemans PJ and Kelleners-Smeets NW: Surgical excision versus Mohs' micrographic surgery for basal cell carcinoma of the face: A randomised clinical trial with 10 year follow-up. Eur J Cancer 50: 3011-3020, 2014.

10. Mastrangelo E and Milani M: Role and inhibition of GLI1 protein in cancer. Lung Cancer (Auck1) 9: 35-43, 2018.

11. Pan D: The hippo signaling pathway in development and cancer. Dev Cell 19: 491-505, 2010.

12. Szelachowska J, Donizy P, Ratajczak-Wielgomas K, Halon A, Zielecka-Debska D, Lichon K, Maciejczyk A, Lata-Wozniak E, Piotrowska A and Matkowski R: The effect of YAP expression in tumor cells and tumor stroma on the prognosis of patients with squamous cell carcinoma of the oral cavity floor and oral surface of the tongue. Oncol Lett 18: 3561-3570, 2019.

13. Quan T, Xu Y, Qin Z, Robichaud P, Betcher S, Calderone K, He T, Johnson TM, Voorhees JJ and Fisher GJ: Elevated YAP and its downstream targets CCN1 and CCN2 in basal cell carcinoma: Impact on keratinocyte proliferation and stromal cell activation. Am J Pathol 184: 937-943, 2014.

14. Chu CY, Chang CC, Prakash E and Kuo ML: Connective tissue growth factor (CTGF) and cancer progression. J Biomed Sci 15: 675-685, 2008 .

15. Hall-Glenn F and Lyons KM: Roles for CCN2 in normal physiological processes. Cell Mol Life Sci 68: 3209-3217, 2011. 
16. Kim NG, Koh E, Chen X and Gumbiner BM: E-cadherin mediates contact inhibition of proliferation through Hippo signaling-pathway components. Proc Natl Acad Sci USA 108: $11930-5,2011$

17. Vornicescu C, Senila SC, Bejinariu NI, Vesa SC, Boșca BA, Chirilă DN, Melincovici CS, Soritău O and Mihu CM: The role of GLI1, YAP, CTGF and E-cadherin in the pathogenesis of basal cell carcinoma - our preliminary results. HVM Bioflux 13: 25-32, 2021

18. Totonchy $M$ and Leffell D: Emerging concepts and recent advances in basal cell carcinoma. F1000Research 6: 2085, 2017.

19. GirardiFM, Wagner VP,Martins MD, Abentroth AL and Hauth LA Factors associated with incomplete surgical margins in basal cell carcinoma of the head and neck. Braz J Otorhinolaryngol: Apr 8 , 2020 (Epub ahead of print). doi: 10.1016/j.bjorl.2020.02.007.

20. Bartoš V, Pokorný D, Zacharová O, Haluska P, Doboszová J, Kullová M, Adamicová K, Péč M and Péč J: Recurrent basal cell carcinoma: A clinicopathological study and evaluation of histomorphological findings in primary and recurrent lesions. Acta Dermatovenerol Alp Pannonica Adriat 20: 67-75, 2011.

21. Saabye Bøgelund F, Ashlede Philipsen P and Gniadecki R: Factors affecting the recurrence rate of basal cell carcinoma. Acta Derm Venereol 87: 330-334, 2007.

22. Nilsson M: Induction of basal cell carcinomas and trichoepitheliomas in mice overexpressing GLI-1. Proc Natl Acad Sci USA 97: 3438-3443, 2000.

23. Cui Y, Cui CA, Yang ZT, Ni WD, Jin Y and Xuan YH: Gli1 expression in cancer stem-like cells predicts poor prognosis in patients with lung squamous cell carcinoma. Exp Mol Pathol 102: 347-353, 2017.

24. Park KS, Martelotto LG, Peifer M, Sos ML, Karnezis AN Mahjoub MR, Bernard K, Conklin JF, Szczepny A, Yuan J, et al: A crucial requirement for Hedgehog signaling in small cell lung cancer. Nat Med 17: 1504-1508, 2011.

25. Debaugnies M, Sánchez-Danés A, Rorive S, Raphaël M Liagre M, Parent M-A, Brisebarre A, Salmon I and Blanpain C: YAP and TAZ are essential for basal and squamous cell carcinoma initiation. EMBO Rep 19: e45809, 2018.

26. Xu MZ, Yao TJ, Lee NP, Ng IO, Chan YT, Zender L, Lowe SW, Poon RT and Luk JM: Yes-associated protein is an independent prognostic marker in hepatocellular carcinoma. Cancer 115 4576-4585, 2009.
27. Guo L, Chen Y, Luo J, Zheng J and Shao G: YAP1 overexpression is associated with poor prognosis of breast cancer patients and induces breast cancer cell growth by inhibiting PTEN. FEBS Open Bio 9: 437-445, 2019.

28. Marx A, Schumann A, Höflmayer D, Bady E, Hube-Magg C, Möller K, Tsourlakis MC, Steurer S,Büscheck F, Eichenauer T, et al: Up regulation of the Hippo signalling effector YAP1 is linked to early biochemical recurrence in prostate cancers. Sci Rep 10: 8916, 2020.

29. Shimbo A, Kajiyama H, Tamauchi S, Yoshikawa N, Ikeda Y, Nishino K, Suzuki S, Niimi K, Sakata J and Kikkawa F: Expression of connective tissue growth factor as a prognostic indicator and its possible involvement in the aggressive properties of epithelial ovarian carcinoma. Oncol Rep 42: 2323-2332, 2019.

30. Lin BR, Chang CC, Che TF, Chen ST, Chen RJC, Yang CY, Jeng YM, Liang JT, Lee PH, Chang KJ, et al: Connective tissue growth factor inhibits metastasis and acts as an independent prognostic marker in colorectal cancer. Gastroenterology 128: 9-23, 2005 .

31. Vanjaka-Rogošić L, Puizina-Ivić N, Mirić L, Rogošić V, Kuzmić-Prusac I, Babić MS, Vuković D and Mardešić S: Matrix metalloproteinases and E-cadherin immunoreactivity in different basal cell carcinoma histological types. Acta Histochem 116: 688-693, 2014

32. Bánkfalvi A, Kraßort M, Buchwalow IB, Végh A, Felszeghy E and Piffkó J: Gains and losses of adhesion molecules (CD44, E-cadherin, and $\beta$-catenin) during oral carcinogenesis and tumour progression. J Pathol 198: 343-351, 2002.

33. Yazdani J, Ghavimi MA, Jabbari Hagh E and Ahmadpour F: The role of E-cadherin as a prognostic biomarker in head and neck squamous carcinoma: A systematic review and meta-analysis. Mol Diagnosis Ther 22: 523-535, 2018.

34. Balci MG and Tayfur M: Loss of E-cadherin expression in recurrent non-invasive urothelial carcinoma of the bladder. Int J Clin Exp Pathol 11: 4163-4168, 2018. International (CC BY-NC-ND 4.0) License. 